PROCEEDINGS OF THE

AMERICAN MATHEMATICAL SOCIETY

Volume 136, Number 12, December 2008, Pages 4329-4338

S 0002-9939(08)09457-4

Article electronically published on June 3, 2008

\title{
AN EXPLICIT SOLUTION OF THE LIPSCHITZ EXTENSION PROBLEM
}

\author{
ADAM M. OBERMAN
}

(Communicated by Walter Craig)

\begin{abstract}
Building Lipschitz extensions of functions is a problem of classical analysis. Extensions are not unique: the classical results of Whitney and McShane provide two explicit examples. In certain cases there exists an optimal extension, which is the solution of an elliptic partial differential equation, the infinity Laplace equation. In this work, we find an explicit formula for a suboptimal extension, which is an improvement over the Whitney and McShane extensions: it can improve the local Lipschitz constant. The formula is found by solving a convex optimization problem for the minimizing extensions at each point. This work extends a previous solution for domains consisting of a finite number of points, which has been used to build convergent numerical schemes for the infinity Laplace equation, and in Image Inpainting applications.
\end{abstract}

\section{INTRODUCTION}

Building Lipschitz extensions of functions is a problem of classical analysis. For functions mapping into the reals, many minimizing extensions exist, and it is desirable to have an optimal one. The optimal extension, at least for Dirichlet data on a domain, is given by the solution of a degenerate elliptic Partial Differential Equation (PDE), the Infinity Laplacian, first studied by Aronsson Aro67. See ACJ04 for a general discussion. This Absolutely Minimizing Lipschitz Extension (AMLE) is optimal in the following sense: repeated application of the extension operator on subdomains does not improve the Lipschitz constant there. AMLEs can be built in the more general setting of metric spaces, provided these are path spaces [Juu02].

The optimal Lipschitz extension problem appears naturally in modern applications. The Lipschitz constant of a mapping between measure spaces gives a measure of the distortion of the mapping [LN05, MST06]. Applications include Image Processing CMS98, and Brain Mapping [MST06. The problem has also been the subject of an interesting connection between differential games and elliptic PDEs [PSSW06].

A key step in the numerical construction of the AMLE used in Obe05 was to build the optimal extension to one additional point, given function values on a finite set of points. This method was adapted to build optimal mappings in MST06; in this case the target space for the application is a two dimensional (cortical) surface, rather than the reals. We also mention the related work [LGA98, LGA96, LG04.

Received by the editors October 22, 2007.

2000 Mathematics Subject Classification. Primary 46A22, 46T20, 58E30, 65D05.

Key words and phrases. Lipschitz extension, absolute minimizers, infinity Laplacian.

(C) 2008 American Mathematical Society Reverts to public domain 28 years from publication 
For mappings into spaces more general than the reals, a classical result in this direction is Kirszbraun's theorem Kir34, which applies to Lipschitz maps from $\mathbb{R}^{n}$ to $\mathbb{R}^{m}$. As explained in [LS97, pg. 2] a key step in building more general extensions of this type is to start with a mapping defined on a finite number of points and to build an extension to one additional point.

On the other hand, for maps with codomain a general metric space (for example $L^{1}$ ), minimizing Lipschitz extensions which do not increase the Lipschitz constant may not always exist. In this case, the question is to study the distortion: the minimal amount that the Lipschitz constant increases [MN06, LN05.

1.1. Contribution of this work. The result which we present here lies in a different direction from the works mentioned above. While those works prove the existence of extensions in a general setting, the goal here is to build an explicit extension.

Let $(X, d)$ be an arbitrary metric space, and let $C$ be a compact subset of $X$. For a given function $g: C \rightarrow \mathbb{R}$, we build a Lipschitz extension to $X$. The extension is sub-optimal (i.e. not an AMLE), but unlike the Whitney and McShane extensions, it can improve the local Lipschitz constant, and it satisfies a maximum principle.

The construction is accomplished as follows. The extension problem from a compact set to one additional point is expressed as a convex optimization problem. An equivalent optimization problem is found which has a saddle point and can be solved explicitly. The solution to the problem at any given point can then be used to define the extension function at all points of the domain directly. The result is a Lipschitz extension function.

The construction extends that of Obe05]. Repeated application of the extension function locally will result in the AMLE Obe05. In the future, we hope to investigate whether the construction can be generalized to other target spaces.

1.2. Whitney and McShane solutions of the Lipschitz extension problem. Given the function $g: C \subset X \rightarrow \mathbb{R}$, the Lipschitz constant $K$ is the least constant for which

$$
\frac{|g(x)-g(y)|}{d(x, y)} \leq K \text { for all } x, y \in C
$$

holds. The Lipschitz extension problem is to build a Lipschitz continuous extension of $g$ with the smallest possible Lipschitz constant.

There are multiple solutions to the Lipschitz extension problem. The Whitney Whi34] and McShane [McS34] extensions,

$$
\bar{u}(x)=\inf _{y \in C}(g(y)+K d(x, y)), \quad \underline{u}(x)=\sup _{y \in C}(g(y)-K d(x, y))
$$

(see Figure 11), are both solutions. In fact they are the maximal and minimal extensions, respectively.

1.3. Examples. We begin by giving some examples of the extension, using notations and definitions which follow.

Example 1. Consider $C \equiv\{-1,+1\} \subset \mathbb{R}$ with $g(-1)=-1, g(+1)=+1$. Refer to Figure 1. The AMLE is not unique in this setting, and the McShane or Whitney extensions do not improve the local Lipschitz constant. In contrast, the extension 

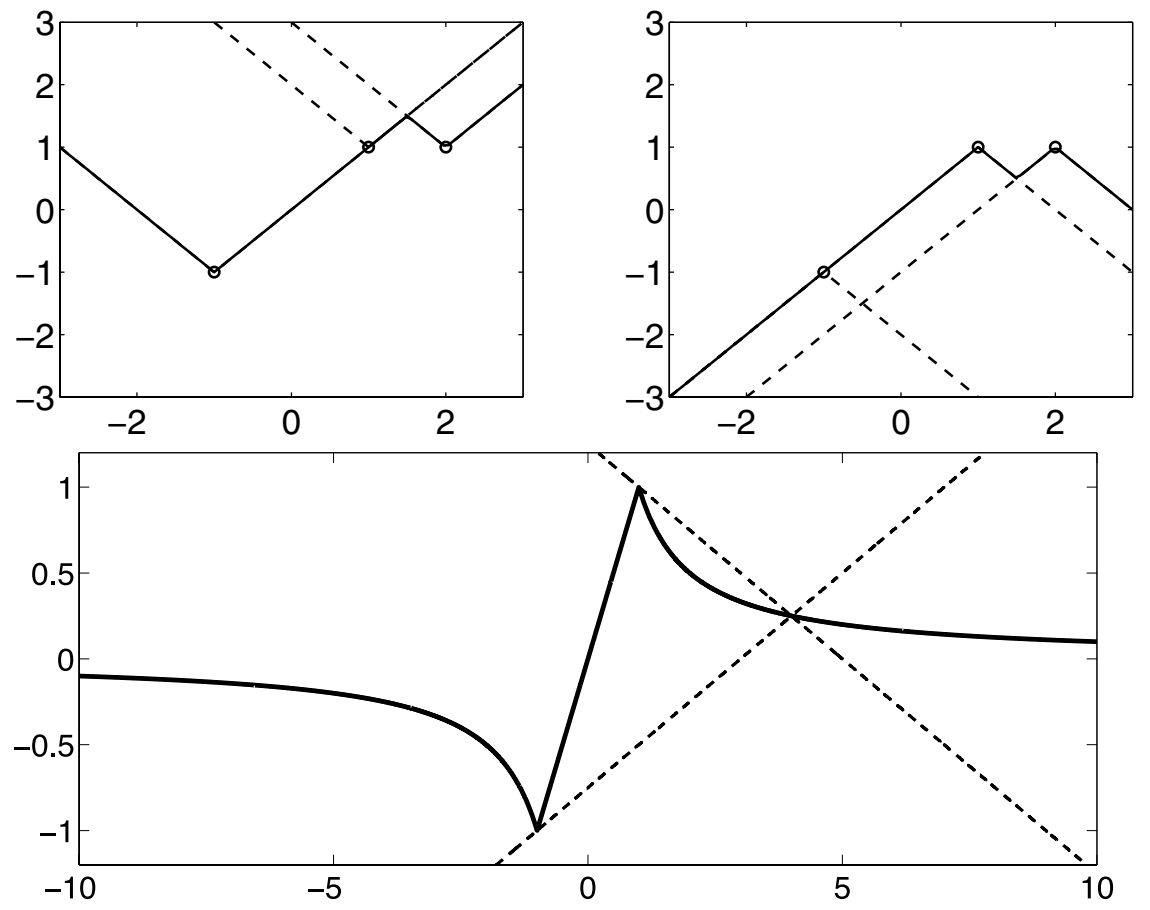

Figure 1. (a) The Whitney and (b) McShane extensions (solid lines) for data consisting of three points (open circles), along with the cones of opening $K$ (dashed lines). (c) The extension $u_{C}(x)$ for data given at two points, and cones at $x$ with opening $K_{C}(x)$.

defined herein is given by

$$
u_{C}(x)= \begin{cases}x & \text { for }|x| \leq 1 \\ \frac{1}{x} & \text { for }|x| \geq 1\end{cases}
$$

and it improves the local Lipschitz constant.

Example 2 (Recovery of smooth solutions of the eikonal equation). Let $v$ be a smooth solution of the eikonal equation $|D v|=c$ in the convex set $\Omega \subset \mathbb{R}^{n}$, with $v=g$ on $\partial \Omega$. Such functions are solutions of the infinity Laplace equation [ACJ04. We claim that $u_{C}(x)=v(x)$. By the the method of characteristics, $v$ is linear on the line $x(t)=x_{0}+t \nabla v\left(x_{0}\right)$. Now let $x_{0} \in \Omega$. We claim that the arg max in (2.5) occurs at the points of intersection of the line $x(t)=x_{0}+t \nabla u_{C}\left(x_{0}\right)$ with $\partial \Omega$. To see this, compute for $w, z \in \partial \Omega$,

$$
D_{z}^{w} g(x)=\frac{|g(w)-g(z)|}{d(w, z)} \frac{d(w, z)}{d(w, x)+d(z, x)} \leq c \frac{d(w, z)}{d(w, x)+d(z, x)} .
$$

The upper bound is obtained when $w, z$ are the endpoints of the line. For such points, (2.6) is the linear interpolant, so the claim is established. 


\section{Definition of the Lipschitz extension}

We define an extension by solving a convex optimization problem at an arbitrary point $x \notin C$. The extension is defined at each point $x$ independently, taking into consideration only the values of the function $g(x)$ on $C$. Collectively, the solutions of each of the problems define an extension function $u_{C}(x)$.

2.1. An optimization problem at each point. The problem of building an extension to the function $g(x)$ defined on the set $C$ at a given point $x \notin C$ involves the unknown value $t=u_{C}(x)$ and the ratio $\frac{|g(y)-t|}{d(y, x)}$, which is the slope of the line from $(x, t)$ to $(y, g(y))$. The goal will be to minimize

$$
J(t ; x) \equiv \max _{y \in C} \frac{|g(y)-t|}{d(y, x)}
$$

the maximum of these slopes, over the values $t$. The function $J$ is convex, since it is a supremum of convex functions. Thus we are led to consider, for $x \in X \backslash C$, the problem

$$
\min _{t} J(t ; x)
$$

whose value and minimizer will be used to define the extension function.

Definition 1. Let $g: C \subset X \rightarrow \mathbb{R}$ be a Lipschitz continuous function on the compact set $C$. Define

$$
K_{C}(x)= \begin{cases}\sup _{y \in C} \frac{|g(y)-g(x)|}{d(x, y)} & \text { for } x \in C, \\ \min _{t} J(t, y ; x) & \text { for } x \in X \backslash C,\end{cases}
$$

and

$$
u_{C}(x)= \begin{cases}g(x) & \text { for } x \in C, \\ \arg \min _{t} J(t, y ; x) & \text { for } x \in X \backslash C .\end{cases}
$$

We establish below that $u_{C}(x)$ is well-defined.

We begin by finding an explicit representation for $u_{C}(x)$. To that end, define

$$
D_{z}^{w} g(x) \equiv \frac{|g(w)-g(z)|}{d(w, x)+d(z, x)}
$$

Theorem 1. Let $g: C \subset X \rightarrow \mathbb{R}$ be a Lipschitz continuous function on the compact set $C$. Then for $x \notin C$,

$$
K_{C}(x)=\max _{(w, z) \in C \times C} D_{z}^{w} g(x),
$$

and for any pair,

$$
\begin{gathered}
\left(x^{+}, x^{-}\right) \in \arg \max _{(w, z) \in C \times C} D_{z}^{w} g(x), \\
u_{C}(x)=\frac{d\left(x^{-}, x\right) g\left(x^{+}\right)+d\left(x^{+}, x\right) g\left(x^{-}\right)}{d\left(x^{-}, x\right)+d\left(x^{+}, x\right)} .
\end{gathered}
$$

So $u_{C}(x)$ is the linear interpolant of the values of $g$ at $x^{+}$and $x^{-}$, weighted by the distances from $x$ to these points. 
2.2. A saddle point proof of Theorem 1. In order to solve (2.1), we relax it in the following way.

Consider for $x \in X \backslash C$ fixed, the function $F: \mathbb{R} \times C \times C \rightarrow \mathbb{R}$ given by

$$
F(t, y, z) \equiv \max \left(\frac{|g(y)-t|}{d(y, x)}, \frac{|g(z)-t|}{d(z, x)}\right),
$$

along with

$$
\bar{J}(t) \equiv \max _{y, z \in C \times C} F(t, y, z)
$$

and the problem

$$
\min _{t} \bar{J}(t) \text {. }
$$

The solution of the relaxed problem (2.8) clearly has the same value as that of the original problem (2.1), but it has a saddle point.

Proof of Theorem 1. Since $x$ is fixed, we drop the $x$-dependence in the notation for the duration of the proof. The resulting notation should be self-explanatory.

We first interchange the minimum and the maximum in (2.8) and solve the problem

$$
\max _{(y, z) \in C \times C} \min _{t} F(t, y, z) .
$$

For fixed $(y, z)$, the solution of $\min _{t} F(t, y, z)$ is given by equating each of the two terms in the maximum (2.7) and occurs when $t^{*}=t^{*}(y, z)$

$$
t^{*}(y, z)=\frac{d(y) g(z)+d(z) g(y)}{d(y)+d(z)}
$$

with

$$
F\left(t^{*}(y, z), y, z\right)=\frac{|g(z)-g(y)|}{d(y)+d(z)} .
$$

Assuming $g(z) \geq g(y)$, plugging (2.9) into (2.10) gives

$$
F\left(t^{*}(y, z), y, z\right)=\frac{g(z)-t^{*}}{d(z)}=\frac{t^{*}-g(y)}{d(y)},
$$

as can be verified by simple arithmetic.

Take

$$
\left(y^{*}, z^{*}\right) \in \arg \max _{(y, z) \in C \times C} F\left(t^{*}(y, z), y, z\right)=\frac{|g(z)-g(y)|}{d(y)+d(z)} .
$$

Then we claim that $\left(t^{*}\left(y^{*}, z^{*}\right), y^{*}, z^{*}\right)$ is a saddle point for $F$; i.e.

$$
F\left(t^{*}, y, z\right) \leq F\left(t^{*}, y^{*}, z^{*}\right) \leq F\left(t, y^{*}, z^{*}\right), \quad \text { for all } t \in \mathbb{R}, y, z \in C .
$$

The second inequality in (2.13) follows immediately since $t^{*}$ is the minimizer. So we only need to prove the first inequality, $F\left(t^{*}, y, z\right) \leq F\left(t^{*}, y^{*}, z^{*}\right)$.

So assume $F\left(t^{*}, y, z\right)>F\left(t^{*}, y^{*}, z^{*}\right)$ for some $y, z$. Follow Figure 2, Without loss of generality, we can assume that the maximum in $F\left(t^{*}, y, z\right)$ occurs with the term involving $y$. First assume that $F\left(t^{*}, y, z\right)=\left(t^{*}-g(y)\right) / d(y)$; the other case will follow using a similar argument. So rewriting our assumption, we have

$$
\frac{t^{*}-g(y)}{d(y)}>\frac{g(z)-t^{*}}{d\left(z^{*}\right)}
$$




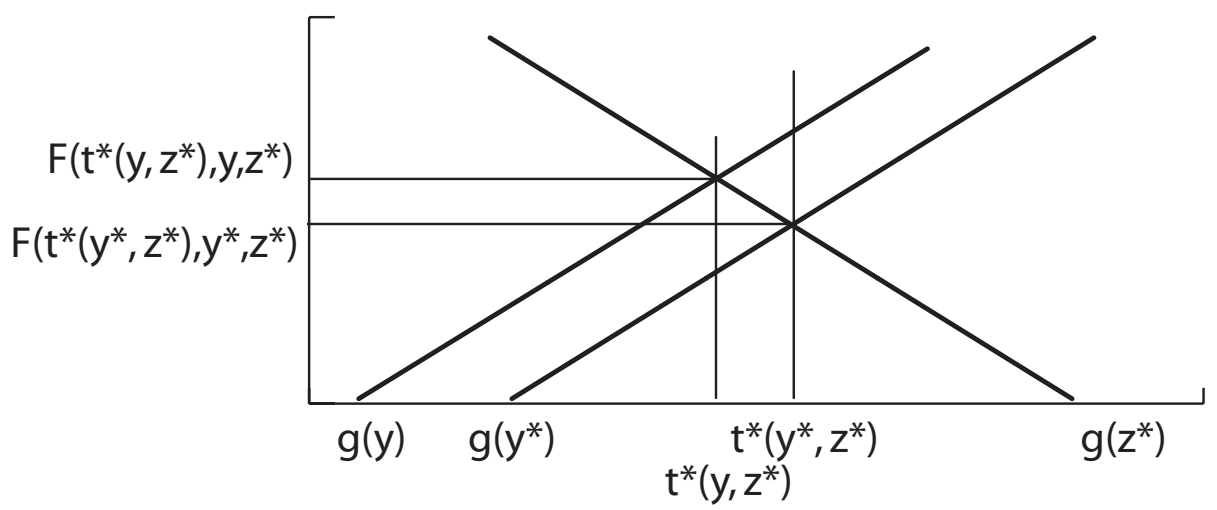

Figure 2. Proof of optimality of $t^{*}$.

where we have used (2.11). Solve for $t^{*}=t^{*}\left(y^{*}, z^{*}\right)$ to get

$$
t^{*}>\frac{d\left(z^{*}\right) g(y)+d(y) g\left(z^{*}\right)}{d\left(z^{*}\right)+d(y)}=t^{*}\left(y, z^{*}\right),
$$

where we have observed that the right-hand side of this last equations corresponds to the optimal $t^{*}\left(y, z^{*}\right)$ as in (2.9). Plugging that value into (2.10) and then using (2.11), we get

$$
F\left(t^{*}\left(y, z^{*}\right), y, z^{*}\right)=\frac{\left|g\left(z^{*}\right)-g(y)\right|}{d(y)+d\left(z^{*}\right)}=\frac{g\left(z^{*}\right)-t^{*}\left(y, z^{*}\right)}{d\left(z^{*}\right)},
$$

but now the inequality in (2.14) gives

$$
\begin{aligned}
\frac{\left|g\left(z^{*}\right)-g(y)\right|}{d(y)+d\left(z^{*}\right)} & =\frac{g\left(z^{*}\right)-t^{*}\left(y, z^{*}\right)}{d\left(z^{*}\right)} \\
& >\frac{g\left(z^{*}\right)-t^{*}\left(y^{*}, z^{*}\right)}{d\left(z^{*}\right)}=\frac{\left|g\left(z^{*}\right)-g\left(y^{*}\right)\right|}{d\left(y^{*}\right)+d\left(z^{*}\right)},
\end{aligned}
$$

which is a contradiction to (2.12). So we have established (2.13).

Remark 1. In the preceding argument, we showed directly that the saddle point gives a solution of the original problem (2.1), so we were justified in interchanging the minimum and the maximum in (2.8).

Standard arguments of convex analysis [Ber03, pp. 131-132] ensure that the existence of the saddle point (2.13) implies

$$
\min _{t} \max _{y, z \in C \times C} F(t, y, z)=\max _{y, z \in C \times C} \min _{t} F(t, y, z) .
$$

2.3. Properties of the extension function. We now establish some basic properties of the extension function.

Theorem 2. Let $C, g(x), u_{C}(x)$ and $K_{C}(x)$ be as in Definition 1. Then

(i) $u_{C}(x)$ is well-defined.

(ii) $u_{C}(x)$ is a continuous extension of $g(x)$.

(iii) (The maximum principle)

$$
\min _{x \in C} g(x) \leq u_{C}(y) \leq \max _{x \in C} g(x) \text { for all } y \in X .
$$


(iv) $0 \leq K_{C}(x) \leq \operatorname{Lip}(g)$ for $x \in X \backslash C$.

(v) $u_{C}(x)$ is Lipschitz continuous, with constant bounded by Lip $(g)$. More precisely,

$$
\frac{\left|u_{C}\left(x_{1}\right)-u_{C}\left(x_{2}\right)\right|}{d\left(x_{1}, x_{2}\right)} \leq \min \left(K_{C}\left(x_{1}\right), K_{C}\left(x_{2}\right)\right), \quad \text { for all } x_{1}, x_{2} \in X \backslash C .
$$

(vi) (Comparison with balanced cones which meet $C$ ) Given $y \in X$, let $c^{ \pm}(x)=$ $u_{C}(y) \pm K(x)|x-y|$ be the upward and downward facing cones which touch $u_{C}$ at $y$. Then

$$
c^{-}(x) \leq u_{C}(x) \leq c^{+}(x) \quad \text { for all } x \in X,
$$

with equality at $x^{-}, x^{+}$, in the first and second inequality, respectively.

(vii) (Growth of $K_{C}$ ) Given $x_{1}, x_{2} \in X \backslash C$,

$$
K_{C}\left(x_{2}\right) \operatorname{dist}\left(x_{2}, C\right) \leq K_{C}\left(x_{1}\right)\left(d\left(x_{1}, x_{2}\right)+\operatorname{dist}\left(x_{2}, C\right)\right) .
$$

Rewrite it as

$$
\frac{K_{C}\left(x_{2}\right)-K_{C}\left(x_{1}\right)}{d\left(x_{1}, x_{2}\right)} \leq \frac{K_{C}\left(x_{1}\right)}{\operatorname{dist}\left(x_{2}, C\right)} .
$$

In particular, when $C=B_{R}$,

$$
K_{C}(x) \leq \frac{1}{1-t} K(0), \quad|x|=t R .
$$

For later use, note from (2.2) that

$$
\left|g(y)-u_{C}(x)\right| \leq K_{C}(x) d(y, x), \quad \text { for all } y \in C,
$$

with equality (choosing labels appropriately) at

$$
\begin{aligned}
& g\left(x^{+}\right)=u_{C}(x)+K_{C}(x) d\left(x^{+}, x\right), \\
& g\left(x^{-}\right)=u_{C}(x)-K_{C}(x) d\left(x^{-}, x\right) .
\end{aligned}
$$

Also we have

$$
K_{C}(x)=D_{x^{-}}^{x^{+}} g(x)=\frac{d\left(x^{+}, x^{-}\right)}{d\left(x^{-}, x\right)+d\left(x^{+}, x\right)} \frac{\left|g\left(x^{+}\right)-g\left(x^{-}\right)\right|}{d\left(x^{+}, x^{-}\right)} .
$$

Proof. Item (iii) follows from (2.6). Item (iv) follows from (2.21).

1. We need to show that (2.1) has a unique minimizer. This can be proved using Danskin's Theorem Ber03, pg. 245], which allows us to compute the directional derivative of the maximum as the maximum of the active directional derivatives. Since the directional derivative of each term of the form $\frac{|g(y)-t|}{d(y, x)}$ is non-zero, the directional derivative of the max is non-zero. Thus the minimizer is unique. However, to keep the argument self-contained, we prove uniqueness directly using the characterization (2.6).

First note that $g$ is defined on a compact set, so the argmax in (2.5) is attained by at least one pair $\left(x^{+}, x^{-}\right)$. Now suppose $\left(x_{1}^{+}, x_{1}^{-}\right)$and $\left(x_{2}^{+}, x_{2}^{-}\right)$are two pairs of points for which the arg max in (2.5) is attained. We wish to show that the corresponding values $u_{1}, u_{2}$, given by (2.6), are equal. So suppose that $u_{1}<u_{2}$. Let $K_{C}(x)$ be as in (2.4), Then, from (2.19), (2.20)

$$
\begin{aligned}
& g\left(x_{2}^{+}\right)=u_{2}(x)+K_{C}(x) d\left(x_{2}^{+}, x\right), \\
& g\left(x_{1}^{-}\right)=u_{1}(x)-K_{C}(x) d\left(x_{1}^{-}, x\right) .
\end{aligned}
$$


But then

$$
D_{x_{1}^{-}}^{x_{2}^{+}} g(x)=\frac{g\left(x_{2}^{+}\right)-g\left(x_{1}^{-}\right)}{d\left(x_{2}^{+}, x\right)+d\left(x_{1}^{-}, x\right)}=K_{C}(x)+\frac{u_{2}(x)-u_{1}(x)}{d\left(x_{2}^{+}, x\right)+d\left(x_{1}^{-}, x\right)},
$$

so we can't have $u_{2}>u_{1}$. A similar argument establishes the reverse inequality. So we have established (ii).

2. Next we establish (iii). Let $x_{0} \in C$, by (2.18). For $x \notin C$, we have

$$
\left|u(x)-g\left(x_{0}\right)\right| \leq K_{C}(x) d\left(x, x_{0}\right) \leq \operatorname{Lip}(g) d\left(x, x_{0}\right),
$$

where we have used (iv). So $u(x) \rightarrow g(x)$ as $C \not \supset x \rightarrow x_{0}$.

3. Next we establish ( $(\mathbf{v})$. Without loss of generality, assume $K_{C}\left(x_{1}\right) \leq K_{C}\left(x_{2}\right)$. Then apply (2.19) to get

$$
g\left(x_{2}^{+}\right)=u_{C}\left(x_{2}\right)+K_{C}\left(x_{2}\right) d\left(x_{2}, x_{2}^{+}\right),
$$

and apply (2.18) with $x=x_{1}$ and $y=x_{2}^{+}$to get

$$
g\left(x_{2}^{+}\right)-u_{C}\left(x_{1}\right) \leq K_{C}\left(x_{1}\right) d\left(x_{2}^{+}, x_{1}\right) .
$$

Combine the two previous equations to get

$$
\begin{aligned}
u_{C}\left(x_{2}\right)-u_{C}\left(x_{1}\right) & \leq K_{C}\left(x_{1}\right) d\left(x_{2}^{+}, x_{1}\right)-K_{C}\left(x_{2}\right) d\left(x_{2}^{+}, x_{1}\right) \\
& \leq K_{C}\left(x_{1}\right)\left(d\left(x_{2}^{+}, x_{1}\right)-d\left(x_{2}^{+}, x_{1}\right)\right) \leq K_{C}\left(x_{1}\right) d\left(x_{1}, x_{2}\right)
\end{aligned}
$$

which gives (2.15), as desired. Finally, applying (iv) gives (v).

4. The main assertion of item (vi) follows from (2.15). The equality conditions follow from (2.19), (2.20).

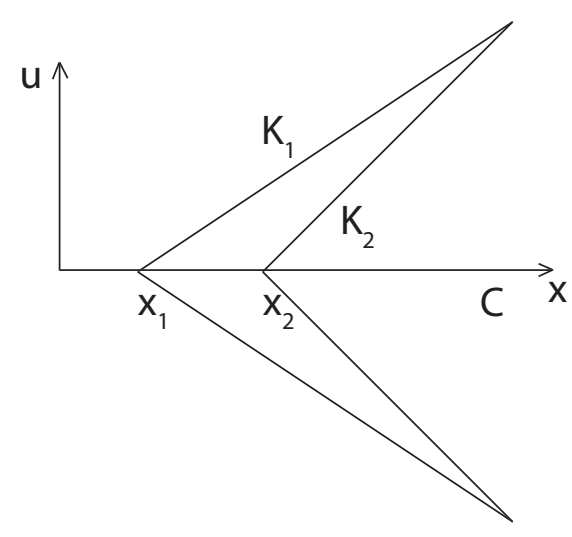

Figure 3. Diagram to illustrate Lipschitz continuity of $K_{C}(x)$ away from $D$.

5. Next we establish (2.16); follow Figure 3. First assume $u_{C}\left(x_{1}\right) \leq u_{C}\left(x_{2}\right)$. Then using (2.19) and the assumption, we have

$$
K_{C}\left(x_{2}\right)=\frac{g\left(x_{2}^{+}\right)-u_{C}\left(x_{2}\right)}{d\left(x_{2}^{+}, x_{2}\right)} \leq \frac{g\left(x_{2}^{+}\right)-u_{C}\left(x_{1}\right)}{d\left(x_{2}^{+}, x_{2}\right)} .
$$

Next, use (2.18) to get

$$
g\left(x_{2}^{+}\right)-u_{C}\left(x_{1}\right) \leq K_{C}\left(x_{1}\right) d\left(x_{2}^{+}, x_{1}\right),
$$


and combine the previous two equations to get

$$
\begin{aligned}
K_{C}\left(x_{2}\right) & \leq K_{C}\left(x_{1}\right) \frac{d\left(x_{2}^{+}, x_{1}\right)}{d\left(x_{2}^{+}, x_{2}\right)} \leq K_{C}\left(x_{1}\right) \frac{d\left(x_{1}, x_{2}\right)+d\left(x_{2}^{+}, x_{2}\right)}{d\left(x_{2}^{+}, x_{2}\right)} \\
& \leq K_{C}\left(x_{1}\right) \frac{d\left(x_{1}, x_{2}\right)+\operatorname{dist}\left(x_{2}, C\right)}{\operatorname{dist}\left(x_{2}, C\right)}
\end{aligned}
$$

which gives (2.16) in this case.

In the case $u_{C}\left(x_{1}\right) \geq u_{C}\left(x_{2}\right)$, we do something similar. Using (2.20) we have

$$
K_{C}\left(x_{2}\right)=\frac{u_{C}\left(x_{2}\right)-g\left(x_{2}^{-}\right)}{d\left(x_{2}^{-}, x_{2}\right)} \leq \frac{u_{C}\left(x_{1}\right)-g\left(x_{2}^{-}\right)}{d\left(x_{2}^{-}, x_{2}\right)},
$$

and (2.18) gives

$$
u_{C}\left(x_{1}\right)-g\left(x_{2}^{-}\right) \leq K_{C}\left(x_{1}\right) d\left(x_{2}^{-}, x_{1}\right) .
$$

The remainder of this case follows as in the previous case.

6. Finally, to get (2.17), take $x_{2}=x,|x|=r, x_{1}=0$ in (2.16) to get

$$
K(x)(R-r) \leq K(0) R
$$

and substitute $r=t R$.

\section{REFERENCES}

[ACJ04] Gunnar Aronsson, Michael G. Crandall, and Petri Juutinen. A tour of the theory of absolutely minimizing functions. Bull. Amer. Math. Soc. (N.S.), 41(4):439-505 (electronic), 2004. MR2083637 (2005k:35159)

[Aro67] Gunnar Aronsson. Extension of functions satisfying Lipschitz conditions. Ark. Mat., 6:551-561, 1967. MR0217665 (36:754)

[Ber03] Dimitri P. Bertsekas. Convex analysis and optimization. Athena Scientific, Belmont, MA, 2003. With Angelia Nedić and Asuman E. Ozdaglar. MR2184037 (2006j:90001)

[CMS98] Vicent Caselles, Jean-Michel Morel, and Catalina Sbert. An axiomatic approach to image interpolation. IEEE Trans. Image Process., 7(3):376-386, 1998. MR.1669524 (2000d:94001)

[Juu02] Petri Juutinen. Absolutely minimizing Lipschitz extensions on a metric space. Ann. Acad. Sci. Fenn. Math., 27(1):57-67, 2002. MR.1884349 (2002m:54020)

[Kir34] M. D. Kirszbraun. Uber die zusammenziehenden und Lipschitzchen Transformationen. Fund. Math., 22:77-108, 1934.

[LG04] E. Le Gruyer. On absolutely minimizing Lipschitz extensions. http://arxiv.org/abs/ math/0403158v1, 2004.

[LGA96] E. Le Gruyer and J. C. Archer. Stability and convergence of extension schemes to continuous functions in general metric spaces. SIAM J. Math. Anal., 27(1):274-285, 1996. MR 1373157 (96k:41002)

[LGA98] E. Le Gruyer and J. C. Archer. Harmonious extensions. SIAM J. Math. Anal., 29(1):279-292 (electronic), 1998. MR.1617186 (99d:54008)

[LN05] James R. Lee and Assaf Naor. Extending Lipschitz functions via random metric partitions. Invent. Math., 160(1):59-95, 2005. MR2129708 (2006c:54013)

[LS97] U. Lang and V. Schroeder. Kirszbraun's theorem and metric spaces of bounded curvature. Geom. Funct. Anal., 7(3):535-560, 1997. MR1466337 (98d:53062)

[McS34] Edward James McShane. Extension of range of functions. Bull. Amer. Math. Soc., 40:837-842, 1934. MR.1562984

[MN06] Manor Mendel and Assaf Naor. Some applications of Ball's extension theorem. Proc. Amer. Math. Soc., 134(9):2577-2584 (electronic), 2006. MR.2213735 (2007a:46014)

[MST06] Facundo Memoli, Guillermo Sapiro, and Paul Thompson. Brain and surface warping via minimizing Lipschitz extensions. IMA Preprint Series 2092, January 2006.

[Obe05] Adam M. Oberman. A convergent difference scheme for the infinity Laplacian: Construction of absolutely minimizing Lipschitz extensions. Math. Comp., 74(251):12171230 (electronic), 2005. MR2137000(2006h:65165) 
[PSSW06] Yuval Peres, O. Schramm, S. Sheffield, and D. Wilson. Tug-of-war and the infinity Laplacian (preprint). http://arxiv.org/PS cache/math/pdf/0605/0605002v1.pdf, 2006.

[Whi34] Hassler Whitney. Analytic extensions of differentiable functions defined in closed sets. Trans. Amer. Math. Soc., 36(1):63-89, 1934. MR.1501735

Department of Mathematics, Simon Fraser University, Burnaby, British Columbia, CANADA V5A 1S6

E-mail address: aoberman@sfu.ca 